Research Article

\title{
Effectiveness of Propofol versus Dexamethasone for Prevention of Postoperative Nausea and Vomiting in Ear, Nose, and Throat Surgery in Tikur Anbessa Specialized Hospital and Yekatit 12th Hospital, Addis Ababa, Ethiopia
}

\author{
Abere Tilahun Bantie $\mathbb{D}^{1},{ }^{1}$ Wosenyeleh Admasu, ${ }^{2}$ Sintayehu Mulugeta, ${ }^{3}$ \\ Abera Regassa Bacha, ${ }^{4}$ and Desalegn Getnet Demsie ${ }^{5}{ }^{5}$ \\ ${ }^{1}$ Department of Anesthesiology, College of Medicine and Health Sciences, Adigrat University, Adigrat, Ethiopia \\ ${ }^{2}$ School of Anesthesiology, College of Health Sciences, Addis Ababa University, Addis Ababa, Ethiopia \\ ${ }^{3}$ Department of Anesthesiology, College of Health Sciences, Mekelle University, Mek'ele, Ethiopia \\ ${ }^{4}$ Department of Anesthesiology, College of Medicine and Health Sciences, Axum University, Axum, Ethiopia \\ ${ }^{5}$ Department of Pharmacy, College of Medicine and Health Sciences, Adigrat University, Adigrat, Ethiopia
}

Correspondence should be addressed to Desalegn Getnet Demsie; desget361@gmail.com

Received 30 April 2020; Revised 7 August 2020; Accepted 21 August 2020; Published 7 September 2020

Academic Editor: Yukio Hayashi

Copyright (C) 2020 Abere Tilahun Bantie et al. This is an open access article distributed under the Creative Commons Attribution License, which permits unrestricted use, distribution, and reproduction in any medium, provided the original work is properly cited.

\begin{abstract}
Background. Postoperative nausea and vomiting (PONV) remain as common and unpleasant and highly distressful experience following ear, nose, and throat surgery. During ENT surgery, the incidence of PONV could be significantly reduced in patients who receive dexamethasone and propofol as prophylaxis. However, the comparative effectiveness of the two drugs has not been assessed. The aim of this study was to compare the effectiveness of propofol and dexamethasone for prevention of PONV in ear, nose, and throat surgery. Methods. This study was conducted in 80 patients, with ASA I and II, aged 18-65 years, and scheduled for ENT surgery between December 20, 2017, and March 20, 2018. Patients were randomly assigned to Group A and Group B. Immediately after the procedure, Group A patients received single dose of intravenous (IV) dexamethasone (10 mg/kg) and Group B patients were given propofol $(0.5 \mathrm{mg} / \mathrm{kg}$, IV), and equal follow-up was employed. The incidence of PONV was noted at 6th, 12th, and 24th hour of drug administration. Independent $t$-test and Mann-Whitney test were used for comparison of symmetric numerical and asymmetric data between groups, respectively. Categorical data were analyzed with the chi-square test, and $p$ value of $<0.05$ was considered as level of significance. Results. The incidences of PONV throughout the 24-hour postoperative period were $35 \%$ in the propofol group and $25 \%$ in the dexamethasone group. Statistical significance was found in incidence of PONV ( $0 \%$ versus $22.5 \%)$ and use of antiemetic ( $0 \%$ versus $5 \%$ ) between dexamethasone and propofol groups, respectively, at 12-24 hours. Over 24 hours, $5 \%$ in dexamethasone group and $12.5 \%$ in propofol group developed moderate PONV, while none of the participants felt severe PONV. Conclusions. Dexamethasone was more effective than propofol to prevent PONV with lower requirements of rescue antiemetics.
\end{abstract}

\section{Introduction}

Postoperative nausea and vomiting (PNOV) remain common and distressing complication following ear, nose, and throat (ENT) surgery, especially when no prophylaxis is given [1-6].
The pathophysiology of PONV is multifactorial; multiple pathways, neurotransmitters, and risk factors are involved. Some of the contributing factors for PONV include age $<50$ years, female gender, history of previous PONV or motion sickness, nonsmoking, obesity, surgical- and anaestheticrelated factors, and/or parental anxiety [7-9]. 
The feelings associated with PONV are unpleasant and distressful requiring multimodal treatment approaches $[7,8]$. Recent evidence indicated that inadequate prevention or treatment of PONV potentiates prolonged recovery and hospitalization, unpleasant hospital experiences, and increased health care costs $[10,11]$. For instance, prolonged vomiting may result in electrolyte imbalance (hypocalcaemia, hypochloremia, and hyponatremic metabolic alkalosis) and dehydration, Mallory-Weis tears, esophageal rupture, aspiration, postoperative bleeding, and airway obstruction especially in patients undergoing ENT surgery [12].

The use of antiemetics can reduce the occurrence of PONV from over $52 \%$ to less than $30 \%$ in certain populations $[1,13]$. To decrease the incidence of PONV, a number of antiemetics including antihistamines [14], butyrophenones [14, 15], serotonin receptor antagonists, corticosteroids [16], and anaesthetic agents [17] have been tried in clinical use. Nevertheless, most of the antiemetics are associated with undesirable adverse effects, such as sedation, hypotension, dysphoria, dry mouth, restlessness, and extrapyramidal symptoms.

Several studies have shown that dexamethasone, a corticosteroid, is an effective antiemetic for PONV prophylaxis in various types of surgery and in improving surgical outcomes $[18,19]$. Propofol, an antagonist at the 5HT3 receptor, is also a novel total intravenous anaesthetic that possesses antiemetic properties when given in subhypnotic doses as part of combination therapy $[5,20]$. Lowdose intravenous propofol $(0.5 \mathrm{mg} / \mathrm{kg})$ is effective for prevention of PONV with no significant complications $[4,21,22]$. Even though propofol has been used by a number of anesthesiologists, it is still under investigation. Thus, the aim of this study was to compare the effectiveness of dexamethasone and propofol for the prevention of PONV in ENT surgery.

\section{Methods}

Ethical approval was obtained from Addis Ababa University, Ethics Committee, and assigned an ethical approval, No-98/ 2010 on December 11, 2018. All patients who were scheduled to undergo elective ENT surgery at Tikur Anbessa Specialized Hospital (TASH) and Yekatit Hospital from December 20, 2017, to March 30, 2018, were enrolled in the study.

All patients of both sexes who had elective ENT surgery under general anesthesia, with ASA I and II, and aged between 18 and 65 years were included in the study. Patients requiring admission to the intensive care unit or mechanical ventilation, premedicated with emetogenic or antiemetic agents, with previous history of nausea/vomiting, with hypotension, gastroesophageal reflux disorder, and insulin-dependent diabetes, and smoking were excluded from the study. Two-independent-sample size formula was used to determine the sample size for each group based on the mean difference of the visual analog score. A total of 80 patients with an American Society of Anesthesiologists physical status I and II and age of 18-65

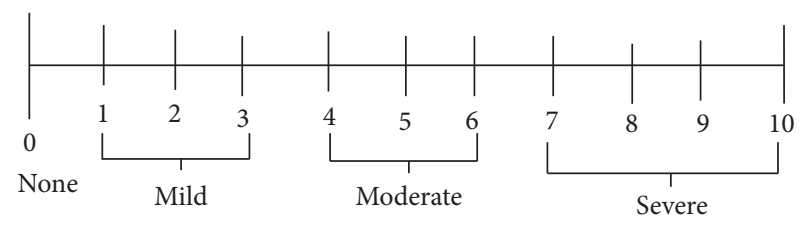

FIgURE 1: Numeric rating scale (NRS).

years were assigned to each group. Afterwards, patients were assigned to either Group A (dexamethasone, $n=40$ ) or Group B (propofol, $n=40$ ) randomly by lottery method from daily schedule list.

Following preoperative preparation, all elective ENT surgery scheduled patients who fulfilled inclusion criteria and volunteered to take part in the study were oriented by trained data collectors on how to self-report nausea using the eleven-point numeric rating score (NRS) from score 0 to 10. Bachelor's and master's anesthetists were responsible for carrying out all anesthesia management. After checking baseline vital signs and achieving adequate preoxygenation for five minutes, patients in both Group A and Group B were induced with intravenous $3-5 \mathrm{mg} / \mathrm{kg}$ thiopentone, $2 \mu \mathrm{g} / \mathrm{kg}$ fentanyl, and $2 \mathrm{mg} / \mathrm{kg}$ suxamethonium. Then anesthesia was maintained with $0.75-1.5 \%$ of halothane with $4 \mathrm{~L} / \mathrm{min}$ flow of $100 \%$ oxygen and intermittent vecuronium $(0.04 \mathrm{mg} / \mathrm{kg})$. At the end of the procedure, patients were fully reversed with $0.02 \mathrm{mg} / \mathrm{kg}$ atropine and $0.04 \mathrm{mg} / \mathrm{kg}$ neostigmine. Immediately after extubation, Group A patients received a single dose of intravenous (IV) $8 \mathrm{mg}$ dexamethasone, while Group B patients were administered with subhypnotic dose of propofol $(0.5 \mathrm{mg} / \mathrm{kg}, \mathrm{IV})$.

Thereafter, in the postanesthesia care unit (PACU), patients were asked to report the severity and occurrence of nausea or vomiting, as well as their need for additional antiemetics based on the 11-point NRS score, once fully able to respond to verbal commands. Patients were fully aware to classify PONV severity as no for score 0 , mild for 1-3, moderate for 4-6, and severe for 7-10 with propensity to vomiting (Figure 1). Then trained professionals had assessed and recorded the severity score (Figure 1). Rescue antiemetic was given at recommended dose intravenously to the patients during active vomiting or with NRS score of 4 and above. The incidence and severity of PONV and associated adverse effects were documented at the 6th hour, 12th hour, and 24th hour after the administration of dexamethasone and propofol. In addition, the requirement of rescue antiemetics in the overall 24 hours was documented.

Statistical analysis was done using SPSS version 20 software. Data distributions were tested by using Shapiro-Wilk test while homogeneity of variance was assessed with Levene's test for equality of variance. Unpaired Student's $t$ test and Mann-Whitney test were used for comparison of numerical variables between study groups. Frequency and percentage were used to describe categorical variables, and the statistical difference between groups was tested using the chi-square test. A $p$ value $<0.05$ with $95 \%$ confidence interval and a power of $80 \%$ were considered statistically significant. 


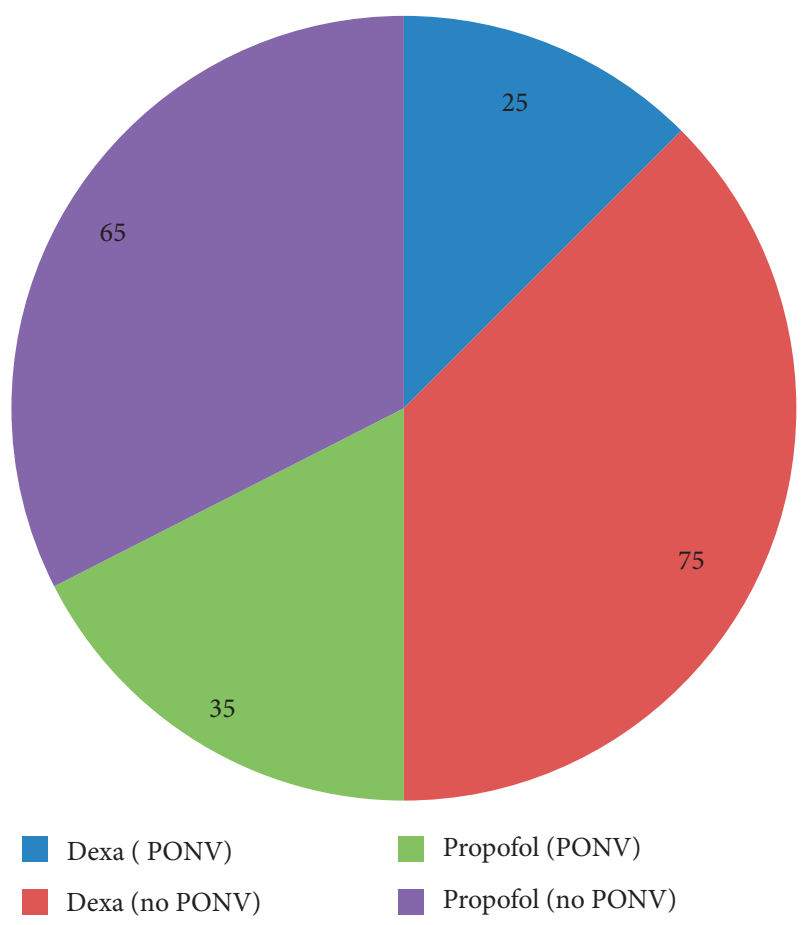

Figure 2: Incidence of PONV (\%) from 0 to 24 hours. Dexa: dexamethasone; PONV: postoperative nausea and vomiting.

\section{Results}

3.1. Sociodemographics and Preoperative Characteristics. The majority of patients were ASA I (82.5\%) and females $(51.25 \%)$. There was no statistically significant difference between the dexamethasone and propofol groups in terms of age, oral intake times, sex, body mass index (BMI), type of surgery, ASA status, or duration of anesthesia and surgery (Table 1).

3.2. Intraoperative Characteristics. Thiopentone (55\%) and both tramadol and diclofenac (48.75\%) were the most commonly used induction analgesic agents, respectively, with no statistically significant difference in the intraoperative variables between the two groups (Table 2).

\subsection{Incidence of PONV and Rescue Antiemetic Use over the} Follow-Up Period. In the dexamethasone group, the requirement of rescue antiemetic treatment ( $0 \%$ versus $5 \%$, $p=0.02)$ and incidence of PONV (0\% versus $22.5 \%$, $p \leq 0.001)$ were statistically significantly lower compared to patients enrolled in propofol group over the 12th-24th hours (Table 3). As indicated in Figure 2, the overall incidence of PONV was higher in patients administered with propofol than those administered with dexamethasone. In propofol group, $35 \%$ of the cases experience PONV, while the incidence of PONV was $25 \%$ in dexamethasone group.

3.4. Severity of Nausea. The incidence of nausea in dexamethasone group gradually decreased in the subsequent time intervals over the 24-hour follow-up period. Of all

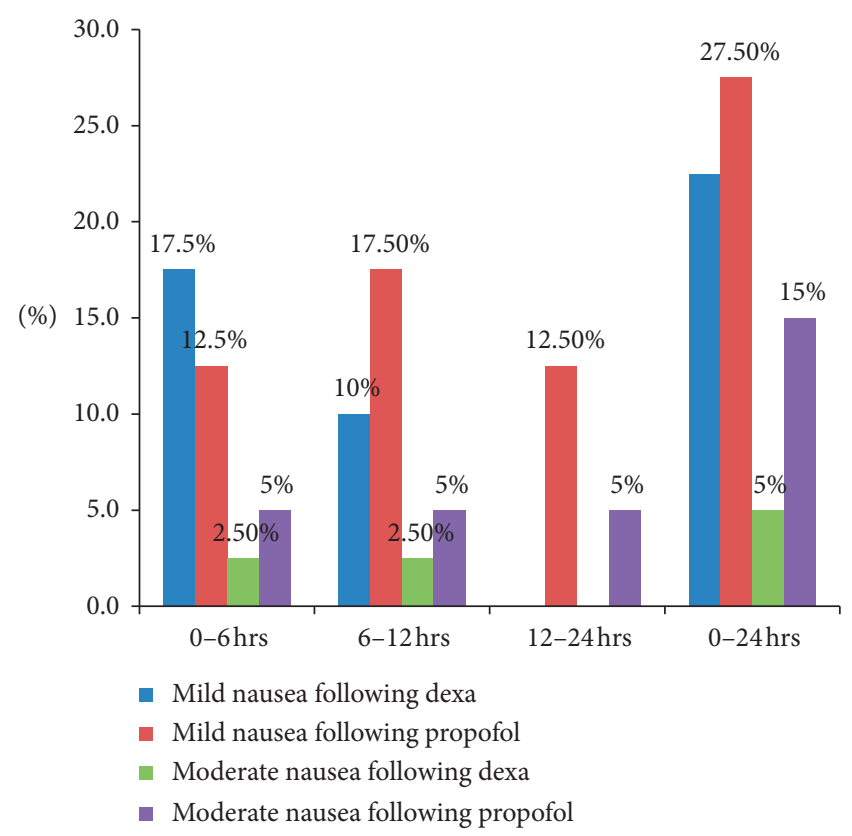

FIgURE 3: Comparison of postoperative nausea severity using 11point NRS score $(0-10)$.

participants, $11(27.5 \%)$ of those receiving propofol reported mild nausea as opposed to $9(22.5 \%)$ of those receiving dexamethasone, while $6(15 \%)$ of propofol recipients experienced moderate nausea compared with $2(5 \%)$ of their dexamethasone receiving counterparts. None of the participants experienced severe nausea in the overall 24-hour follow-up period. These findings were statistically significant in the $12-24 \mathrm{hr}$ postoperative period $(p=0.012)$ (Figure 3 ).

3.5. Reported Side Effects. During the overall follow-up, $2.5 \%$ of patients in propofol group and 5\% patients in dexamethasone group complained of dizziness, whilst 3\% of patients in propofol group reported sedation, but there was no hypoxia and difficulty of breathing and $2.5 \%$ of participants in the dexamethasone group experienced headache (Figure 4).

\section{Discussion}

ENT surgery has been associated with high incidence of PONV, especially in patients without prophylactic antiemetic agents $[2,4,23]$. During a surgical procedure, serotonin is released from the gastrointestinal tract from enterochromaffin cells and binds to visceral receptors of the 5-HT 3 subtype, causing stimulation of vagal afferents in the gastrointestinal tract to conduct impulses that reach the Chemoreceptor Trigger Zone (CTZ) located on the dorsal surface of the medulla oblongata at the caudal end of the fourth ventricle. CTZ stimulation due to the arrived stimulus will lead to PONV [24].

In our study, the overall incidence of PONV was higher in the propofol group than dexamethasone group (35\% versus $25 \%$ ) with statistical significance in the 12 th to 24 th 


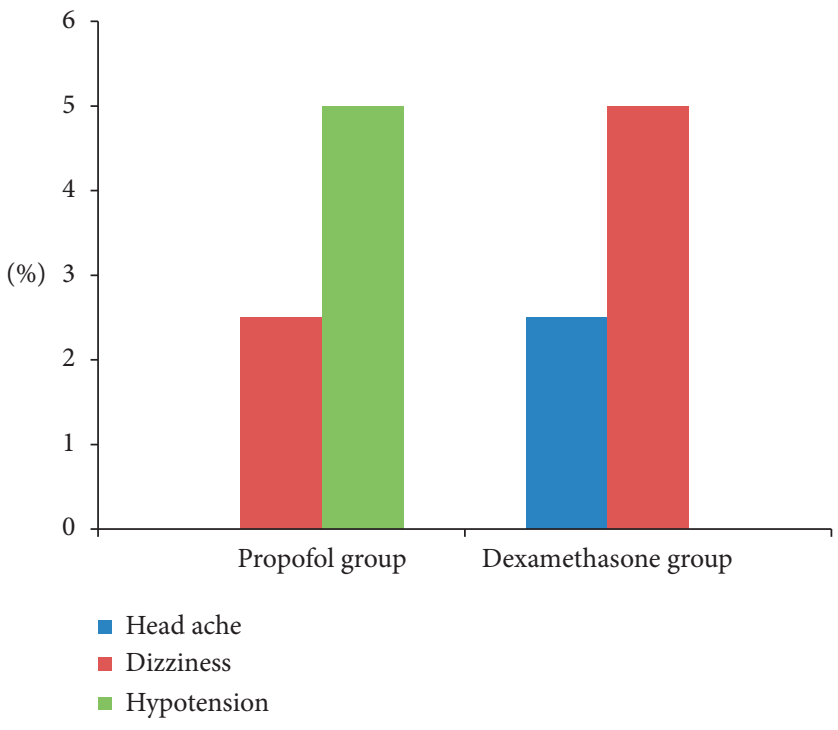

Figure 4: Associated side effects observed within 24 hours after taking antiemetics.

hour period $(p<0.002)$. The requirement for rescue antiemetics was relatively lower in dexamethasone group.

Glucocorticoids have been widely used to prevent PONV during chemotherapy use or general anesthesia. Although the antiemetic mechanism is not clearly understood, scientific evidence suggests that dexamethasone reduces production and release of 5-HT and decreases permeability across the Blood-Brain Barrier (BBB) thereby lowering the amount of 5-HT available to chemical sensors [18, 25]. However, the use of dexamethasone may be associated with increased risk of infection, reduced wound healing, and interference with the functioning of adrenal glands through negative feedback-mediated reduction of endogenous steroid synthesis.

In dexamethasone group, the incidence of PONV was low in the periods of $0-6 \mathrm{hrs}$ and $6-12 \mathrm{hrs}$, while there are no participants encountering nausea and vomiting in the latter period (i.e., 12-24hrs). Indeed, low or no PONV with dexamethasone could be attributable to its fast onset and long duration of action (i.e., 72 hours) [26].

Similarly, a bolus dose of propofol produced preventive antiemetic effects in patients undergoing ENT surgery in this study. However, the protective action of propofol was decreased in the subsequent time intervals as compared to dexamethasone group. In line with this result, the requirement of rescue antiemetic therapy was lower in the dexamethasone than propofol recipients $(5 \%$ versus $12.5 \%, p<0.23)$ over the $24 \mathrm{hr}$ period. Statistically significant results were obtained at the 12th to 24th hour time period $(p<0.044)$. Studies suggest that effective concentration of propofol is better maintained in IV infusion rather than bolus dosing to prevent PONV $[27,28]$. The antiemetic effect of propofol is attributed to modulation of subcortical pathways to inhibit nausea or its direct depressant action on the vomiting center [29]. The results of our study are consistent with other studies, conducted in different settings, in terms of antiemetic rescue therapy requirements and trends of dexamethasone preventive effect $[22,23,25,30,31]$.

\section{Conclusion}

In summary, dexamethasone produced better PONV protection than propofol in all time intervals. Nevertheless, there are some limitations in this study. For instance, values were not statistically significant in most of the time intervals, which may reflect the small sample size in both groups. Moreover, placebos were not used to check the outcomes of drug treatment and without treatment. Therefore, we recommend future research with a larger sample size and studies with placebo group. Further, we recommend a randomized controlled trial be conducted to further validate these findings.

\section{Abbreviations \\ ASA: American Society of Anesthesiology \\ BMI: Body mass index \\ ENT: Ear, nose, and throat \\ 5HT3: 5-Hydroxytryptamine receptors \\ ICU: Intensive care unit \\ NRS: Numeric rating score \\ PONV: Postoperative nausea and vomiting.}

\section{Data Availability}

The data for this study were collected from Tikur Anbessa Specialized Hospital and Yekatit 12th Hospital. The collected data are available in SPSS and data collection tools.

\section{Ethical Approval}

Ethical clearance and approval were obtained from the ethical review committee, Addis Ababa University College of Health Sciences. Permission to conduct was obtained from Tikur Anbessa Specialized and Teaching Hospital and Yekatit 12th Hospital.

\section{Consent}

The purpose of the study was explained to the patients under the study and written informed consent was obtained. The patients were informed that the care to be given would not be compromised in any way and confidentiality was assured. Name and other pieces of identifying information were not used in the study.

\section{Conflicts of Interest}

The authors declare that they have no conflicts of interest.

\section{Authors' Contributions}

Abere Tilahun Bantie designed the study, supervised the data collection, performed the analysis, interpreted data, drafted the manuscript, and obtained final approval of the revision for publication. Wosenyeleh Admasu and Bacha Abera 
Regassa assisted in designing the study and data interpretation and critically reviewed the manuscript. Sintayehu Mulugeta assisted in designing the study, data analysis, and data interpretation and reviewed the manuscript critically. Desalegn Getnet Demsie assisted in designing the study, analysis, methodology, and interpretation of the data with the statistics and reviewed the manuscript critically. The agreement is accountable for all aspects of the work in ensuring that questions related to the accuracy or integrity of the work are appropriately investigated and resolved. All authors also read and approved the final manuscript.

\section{Acknowledgments}

The authors would like to acknowledge Addis Ababa University, the supervisors, data collectors, and study participants for their invaluable support. Special gratitude goes to Pammla Petrucka (RN, BScN, MN, PhD), Professor at University of Saskatchewan, for her immense support in manuscript preparation.

\section{Supplementary Materials}

Table 1: sociodemographics and preoperative characteristics of study participants. Table 2: intraoperative characteristics of participants. Table 3: incidence of PONV and requirement of rescue antiemetic. (Supplementary Materials)

\section{References}

[1] P. Ewalenko, S. Janny, M. Dejonckheere, G. Andry, and C. Wyns, "Antiemetic effect of subhypnotic doses of propofol after thyroidectomy," British Journal of Anaesthesia, vol. 77, no. 4, pp. 463-467, 1996.

[2] C. L. Burlacu, D. Healy, D. J. Buggy, C. Twomey, D. Veerasingam, and A. Tierney, "Continuous gastric decompression for postoperative nausea and vomiting after coronary revascularization surgery," Anesthesia \& Analgesia, vol. 100 , no. 2 , pp. 321-326, 2000.

[3] T. J. Gan, "Risk factors for postoperative nausea and vomiting," Anesthesia \& Analgesia, vol. 102, no. 6, pp. 1884-1898, 2006.

[4] C. C. Apfel, E. Läärä, M. Koivuranta, C.-A. Greim, and N. Roewer, "A simplified risk score for predicting postoperative nausea and vomiting," Anesthesiology, vol. 91, no. 3, p. $693,1999$.

[5] H. Matsuura, S. Inoue, and M. Kawaguchi, "The risk of postoperative nausea and vomiting between surgical patients received propofol and sevoflurane anesthesia: a matched study," Acta Anaesthesiologica Taiwanica, vol. 54, no. 4, pp. 114-120, 2016.

[6] G. Dewinter, A. Teunkens, K. Vermeulen et al., "Alizapride and ondansetron for the prevention of postoperative nausea and vomiting in patients undergoing laparoscopic gynaecological surgery," European Journal of Anaesthesiology, vol. 33, no. 2, pp. 96-103, 2016.

[7] C. Apfel, P. Kranke, and L. Eberhart, "Comparison of surgical site and patient's history with a simplified risk score for the prediction of postoperative nausea and vomiting," Anaesthesia, vol. 59, no. 11, pp. 1078-1082, 2011.
[8] X. Cao, P. F. White, and H. Ma, "An update on the management of postoperative nausea and vomiting," Journal of Anesthesia, vol. 31, no. 4, pp. 617-626, 2017.

[9] S. I. Shaikh, D. Nagarekha, G. Hegade, and M. Marutheesh, "Postoperative nausea and vomiting: a simple yet complex problem," Anesthesia, Essays and Researches, vol. 10, no. 3, p. 388, 2016.

[10] A. L. Kovac, "Prevention and treatment of postoperative nausea and vomiting," Drugs, vol. 59, no. 2, pp. 213-243, 2000.

[11] T. J. Gan, K. G. Belani, S Bergese et al., "Fourth consensus guidelines for the management of postoperative nausea and vomiting," Anesthesia \& Analgesia, vol. 131, no. 2, pp. 411448, 2019.

[12] Y. Unal, O. Ozsoylar, M. Arslan, D. Sarigüney, and M. Akçabay, "Comparison of the efficacy of propofol and metoclopramide in preventing postoperative nausea and vomiting after middle ear surgery," Saudi Medical Journal, vol. 30, no. 6, pp. 778-782, 2009.

[13] J. Mosley, "The effectiveness of a preoperative multimodal antiemetic regimen on reducing early postoperative nausea and vomiting in total joint arthroplasty patients," 2015.

[14] S. Mehrotra, "Postoperative anaesthetic concerns in children: postoperative pain, emergence delirium and postoperative nausea and vomiting," Indian Journal of Anaesthesia, vol. 63, no. 9, p. 763, 2019.

[15] P. Ziemann-Gimmel, R. Schumann, W. English, J. Morton, and A. Wadhwa, "Preventing nausea and vomiting after bariatric surgery: is the apfel risk prediction score enough to guide prophylaxis?” Obesity Surgery, 2020.

[16] L. Nordin, A. Nordlund, A. Lindqvist, H. Gislason, and J. L. Hedenbro, "Corticosteroids or not for postoperative nausea: a double-blinded randomized study," Journal of Gastrointestinal Surgery, vol. 20, no. 8, pp. 1517-1522, 2018.

[17] M. Chubsaz, M. Rezaei, A. Lahoorpour, and R. M. Jafari, "Dexmedetomidine versus Propofol in reducing postoperative nausea and vomiting in gynecologic laparoscopic surgery," Journal of Kermanshah University of Medical Sciences (Behbood), vol. 21, no. 2, pp. 51-56, 2017.

[18] J.-J. Wang, S.-T. Ho, S.-C. Lee, Y.-C. Liu, and C.-M. Ho, "The use of dexamethasone for preventing postoperative nausea and vomiting in females undergoing thyroidectomy: a doseranging study," Anesthesia \& Analgesia, vol. 91, no. 6, pp. 1404-1407, 2000.

[19] Dreams Trial Collaborators and West Midlands Research Collaborative, "Dexamethasone versus standard treatment for postoperative nausea and vomiting in gastrointestinal surgery: randomised controlled trial (DREAMS Trial)," $B M J$, vol. 18, p. 357, 2017.

[20] D. Song, C. W. Whitten, P. F. White, S. Y. Yu, and E. Zarate, "Antiemetic activity of propofol after sevoflurane and desflurane anesthesia for outpatient laparoscopic cholecystectomy," Anesthesiology, vol. 89, no. 4, pp. 838-843, 1998.

[21] K. Firdaus, A. Dan, M. Maaya et al., "Dexamethasone $8 \mathrm{mg}$ versus dexamethasone $4 \mathrm{mg}$ with propofol $0.5 \mathrm{mg} / \mathrm{kg}$ for the prevention of postoperative nausea and vomiting after laparoscopic gynaecology procedure," International Medical Journal, vol. 23, no. 1, 2016.

[22] Ö. ÖzmeN, F. BİngÖl, Z. ARslAn, and B. Ö. BİngÖl, "The effects of dexamethasone vs low doses of propofol infusion on postoperative nausea and vomiting in tympanoplasty surgery: a randomized, placebo-controlled, double-blinded study," Medeniyet Medical Journal, vol. 32, no. 2, pp. 85-90, 2017.

[23] M. Celik, A. Dostbil, M. Aksoy, I. Ince, A. Ahiskalioglu, and M. Comez, "Is infusion of subhypnotic propofol as effective as 
dexamethasone in prevention of post operative nausea and vomiting related to laparoscopic cholecystectomy? A randomized controlled trial," BioMed Research International, vol. 2015, 2015.

[24] T. J. Gan, "Mechanisms underlying postoperative nausea and vomiting and neurotransmitter receptor antagonist-based pharmacotherapy," CNS Drugs, vol. 21, no. 10, pp. 813-833, 2007.

[25] C. Zhou, Y. Zhu, Z. Liu, and L. Ruan, "5HT3 antagonists versus dexamethasone in the prevention of PONV in patients undergoing laparoscopic cholecystectomy: a meta-analysis of RCTs," BioMed Research International, vol. 2016, 2016.

[26] https://davisplus.fadavis.com/3976/meddeck/pdf/ dexamethasone.pdf.

[27] A. J. Soppitt, P. S. A. Glass, S. Howell, K. Weatherwax, and T. J. Gan, "The use of propofol for its antiemetic effect: a survey of clinical practice in the United States," Journal of Clinical Anesthesia, vol. 12, no. 4, pp. 265-269, 2000.

[28] H. Yimer, N. Ayalew, Z. Abdisa, and A. Aregawi, "Effect of sub-hypnotic dose of propofol on prevention of postoperative nausea and vomiting as part of multimodal antiemetic in patients undergoing open abdominal surgery: a prospective cohort study, Gondar University Hospital, Northwest Ethiopia, 2016," International Journal of Surgery Open, vol. 10, pp. 15-20, 2018.

[29] https://anesthesiageneral.com/propofol-antiemetic/.

[30] A. M. DM, "Prevention of post operative nausea and vomiting with small dose of propofol and dexamethasone in a patient undergoing ENT surgery," Bratisl Lek Listy, vol. 12, no. 6, pp. 332-336, 2011.

[31] Y. Fujii and M. Nakayama, "Retracted article: prevention of postoperative nausea and vomiting with a small dose of propofol alone and combined with dexamethasone in patients undergoing laparoscopic cholecystectomy: a prospective, randomized, double-blind study," Surgical Endoscopy, vol. 22, no. 5, pp. 1268-1271, 2008. 